\title{
Grounding after moderate eccentric contractions reduces muscle damage
}

This article was published in the following Dove Press journal:

Open Access Journal of Sports Medicine

21 September 2015

Number of times this article has been viewed

\section{Richard Brown' \\ Gaétan Chevalier ${ }^{2}$ \\ Michael Hill ${ }^{3}$}

'Department of Human Physiology, University of Oregon, Eugene, OR, ${ }^{2}$ Developmental and Cell Biology Department, University of California at Irvine, Irvine, CA, ${ }^{3}$ Garden Way Chiropractic Center and Sports Injury Clinic, Eugene, OR, USA
Correspondence: Gaétan Chevalier Developmental and Cell Biology Department, University of California at Irvine, 2108 McGaugh Hall, Irvine, CA $92697-2300$, USA

$\mathrm{Tel}+\mathrm{I} 760815$ 927|

Fax + I 85822535 I4

Email dlbogc@sbcglobal.net
Abstract: Grounding a human to the earth has resulted in changes in the physiology of the body. A pilot study on grounding and eccentric contractions demonstrated shortened duration of pain, reduced creatine kinase (CK), and differences in blood parameters. This follow-up study was conducted to investigate the effects of grounding after moderate eccentric contractions on pain, CK, and complete blood counts. Thirty-two healthy young men were randomly divided into grounded $(n=16)$ and sham-grounded $(n=16)$ groups. On days 1 through 4 , visual analog scale for pain evaluations and blood draws were accomplished. On day 1, the participants performed eccentric contractions of 200 half-knee bends. They were then grounded or sham-grounded to the earth for 4 hours on days 1 and 2. Both groups experienced pain on all posttest days. On day 2, the sham-grounded group experienced significant $\mathrm{CK}$ increase $(P<0.01)$ while the $\mathrm{CK}$ of the grounded group did not increase significantly; the between-group difference was significant $(P=0.04)$. There was also an increase in the neutrophils of the grounded group on day $3(P=0.05)$ compared to the sham-grounded group. There was a significant increase in platelets in the grounded group on days 2 through 4 . Grounding produced changes in $\mathrm{CK}$ and complete blood counts that were not shared by the sham-grounded group. Grounding significantly reduced the loss of CK from the injured muscles indicating reduced muscle damage. These results warrant further study on the effects of earthing on delayed onset muscle damage.

Keywords: creatine kinase, free radicals, neutrophils, platelets, reactive oxygen species, ROS

\section{Introduction}

Until recently, humans lived in direct bare skin contact with the surface of the Earth. Modern life has insulated us from this connection. The surface of Earth has a negative electric charge that is maintained by a global electric circuit created mainly by thousands of daily lightning strikes. ${ }^{1,2}$ Electrons from the lightning enter the Earth's surface making it an electrical reference point with an electric potential set to $0 \mathrm{~V}$ by universal agreement. A person in direct skin contact with the Earth's surface has the same electric potential as the Earth. Contact with the Earth can also be attained through the use of conductive sheets, mats, bands, or electrode patches connected to the Earth's surface. This connection enables transfer of the Earth's free electrons into the electrically conductive human body. ${ }^{3}$

When insulated from the earth outdoors, our body equilibrates with the electrically positive atmosphere. Additionally, when we are insulated from the earth indoors, our body becomes electrically energized by ambient electromagnetic fields from wires, lights, and appliances. This ungrounded state may disrupt the natural functioning of the body's electric systems. ${ }^{4}$ 
When insulated indoors, alternating current voltage induced on the body can be $3.50 \mathrm{~V}$ or higher. Because the body is a semiconductor in an aqueous solution, ${ }^{5}$ unnatural charges could interfere with the normal function of human processes. But when we are grounded to the Earth, our electric potential is close to zero. ${ }^{3,6}$ Grounding reduces the electrostatic charge on the body and returns it to Earth's potential. ${ }^{67} \mathrm{In}$ an insulated environment, having an unlimited source of free electrons via grounding could play a regulatory and restorative role of clinical significance. ${ }^{8}$

Peer-reviewed studies have demonstrated beneficial effects of grounding. Benefits include: decreased blood urea concentrations during exercise; ${ }^{9}$ increased surface charge on red blood cells and decreased aggregation; ${ }^{4}$ improved heart rate variability; ${ }^{10}$ decreased night-time levels of cortisol; ${ }^{11}$ and decreased blood oxygenation variances. ${ }^{12}$ A pilot study that created delayed-onset muscle soreness (DOMS) via eccentric contractions pointed toward a positive alteration in the immune system and reduction of pain after grounding. ${ }^{13}$ Parameters that differed by $10 \%$ or more included CK, white blood cells, neutrophils, bilirubin, inorganic phosphate/phosphocreatine ratios ( $\mathrm{Pi} / \mathrm{PCr})$, and pain scale measurements.

In the present study, we subjected young adult males to moderate DOMS via eccentric/concentric contractions. There is an association between eccentric contractions and muscle damage. ${ }^{14}$ Following eccentric contractions, as well as challenging continuous concentric contractions, skeletal muscle fibers suffer damage related to membrane disruption. ${ }^{15}$ The injuries to the muscle cells result in a condition called DOMS that occurs within 24 hours of the activity. ${ }^{16}$ The structural damage leads to an inflammatory response. ${ }^{15}$ Protocols using machines or weights to produce the damage were not considered because of time, equipment, and financial restrictions.

While free radicals or reactive oxygen species (ROS) are crucial in the reduction of acute inflammation, they also become detrimental if they leak from the site of injury or overwhelm the body's antioxidant defenses via secondary bursts. Rogue ROS create a chain reaction and damage healthy molecules and DNA. Antioxidants can neutralize ROS involved in the inflammatory response. Perhaps electrons from the earth, as an additional source of free electrons acting as antioxidants, can help neutralize rogue ROS and assist in the healing process. ${ }^{17}$

We wanted to determine how grounding would affect various physiological markers associated with the inflammatory process.

\section{Material and methods}

\section{Subjects}

Thirty-two healthy male students from the University of Oregon Human Physiology Department (Eugene, OR, USA) were recruited and randomized to either grounded or sham-grounded groups. All subjects were males between the ages of 18 and 24 years, weighed between 66 and 84 kilograms, and were between 171 and $185 \mathrm{~cm}$ tall. There was no significant difference between groups in age ( $P=0.38)$, height $(P=0.47)$, or weight $(P=0.18)$. Details of subjects' characteristics are presented in Table 1.

They had no surgeries or musculoskeletal problems that would prohibit them from doing 200 knee bends. They were active young men who were active at least 4 days per week for at least an hour per day. None practiced half squats on a consistent basis. Subjects avoided the use of alcohol and made a commitment not to be physically active during the study.

\section{Grounding equipment and method}

The grounding equipment included $5.1 \times 10.2 \mathrm{~cm}$ transcutaneous electrical nerve stimulation-type electrode patches (UltraStim Electrodes, Model SN2040; Axelgaard Manufacturing Co Ltd, Fallbrook, CA, USA), $50.8 \times 30.5 \mathrm{~cm}$ conductive mats, and connecting cords to the ground. A $100 \mathrm{k} \Omega$ resistor was molded into the cords for surge protection. The cords used for sham-grounding were modified so that they prevented connection to the earth (they maintained an open circuit), but that was not apparent and no difference could be seen between functioning grounding cords and sham-grounding cords. Grounding equipment was provided by the sponsor (http://www.earthing.com).

When it was time for subjects to be grounded, they were seated and grounding patches were put on their quadriceps. They placed their bare feet on a grounding mat on the floor. For a period of 4 hours, participants were thus grounded or sham-grounded.

\section{Experimental design}

All study procedures were reviewed and approved by the Western Institutional Review Board in Olympia, WA, USA.

Table I Participant characteristics

\begin{tabular}{llll}
\hline & All & Grounded & Sham-grounded \\
\hline $\mathrm{n}$ & 32 & 16 & 16 \\
Age (years) & $21.2 \pm 1.6$ & $21.1 \pm 1.8$ & $21.3 \pm 1.5$ \\
Height $(\mathrm{cm})$ & $177.7 \pm 8.1$ & $178.4 \pm 5.2$ & $177.0 \pm 10.9$ \\
Weight $(\mathrm{kg})$ & $77.7 \pm 5.2$ & $78.0 \pm 5.8$ & $76.4 \pm 4.7$ \\
BMI $\left(\mathrm{kg} / \mathrm{m}^{2}\right)$ & $25.4 \pm 2.9$ & $24.6 \pm 2.5$ & $26.1 \pm 3.4$ \\
\hline
\end{tabular}

Note: Values are expressed as mean \pm SD.

Abbreviations: BMI, body mass index; SD, standard deviation. 
The study was conducted at the Bowerman Sports Science Clinic at the University of Oregon, Eugene, OR, USA. Subjects were provided with and signed informed consent forms prior to the commencement of the study. Subjects were compensated $\$ 150$ plus grounding equipment for their participation.

For day 1 of the study, the 32 subjects were randomly divided into four groups of eight. They were each given a code number on a yellow or blue card at the blood draw station. Subjects with the yellow card were in the grounded group while subjects with the blue card were sham-grounded. The first group arrived at 9 am and then another group arrived every 36 minutes. Each subject filled in the visual analog scale (VAS) and underwent a blood draw of 9-11 $\mathrm{mL}$ for creatine kinase $(\mathrm{CK})$ analysis via a Beckman AU480 and a complete blood count (CBC) via a Sysmax XT 2000i. The blood was drawn by a certified phlebotomist from Legacy Laboratory Services in Eugene, OR, USA.

Each group then proceeded to the knee bend area in the Bowerman Sports Science Clinic where they performed 200 half-knee bends (thighs parallel to the floor) with a $5.1 \times 10.2 \mathrm{~cm}$ board under their heels to help isolate the quadriceps muscle group. Half-knee bends were chosen because of the extensive literature review, and because the investigator had performed four previous experiments using half-knee bends and attained an increase in CK each time. The exercise had to be completed over a 10-minute period at a cadence of 20 half-knee bends every minute, a pace designed to safely produce mild cellular disruptions in the quadriceps muscle group. When the thighs were parallel to the floor, the position was held for 2 seconds. If subjects could not complete the 200 knee bends completely, they would be eliminated from the study (no subjects were eliminated in this way). The subjects were monitored by three trained observers.

After the exercise, subjects proceeded to a therapy area in the clinic with the yellow or blue card containing their code number. Each subject selected grounding equipment corresponding to his card color. Later, these numbers were transferred to electronic data. Half the grounding equipment was functional and half was sham equipment. Only the sponsor knew which was functional and which was sham until after the study and statistical evaluations were completed. The subjects were seated and connected to grounding patches on their quadriceps. They placed their feet on a grounding mat on the floor. For a period of 4 hours, participants were thus grounded or sham-grounded. They were able to drink approximately $1 \mathrm{~L}$ (32 ounces) of water, go to the bathroom, study, read, and use electronic devices.

After the grounding session, subjects left the clinic. They were instructed to limit their physical activity to walking to class and to avoid alcohol and recreational drugs until after the final blood draw of the study on day 4.

On day 2 , in the same timeframe as day 1 , the participants reported to the clinic starting at $9 \mathrm{am}$. Each filled in the VAS, had a blood draw, and then proceeded to the therapy area and connected to the same grounding patches and mat for a similar period of 4 hours. As before, subjects were able to drink 32 ounces of water, go to the bathroom, study, read, and use electronic devices.

On days 3 and 4 , in the same timeframe as day 1, the participants reported to the clinic where they filled in the VAS and had a blood draw.

\section{Procedures}

The VAS was completed in approximately 1 minute. Each venipuncture; including verification, set-up, blood draw, band aid application, and test tube placement in a cold box took approximately 4 minutes per subject. Each subject had a total of four venipunctures over 4 days.

The knee bends took 10 minutes and were preceded by a 5-minute warm-up period following 5 minutes of instructions. Total time for the eccentric contraction portion of the study was 20 minutes.

The next part of the study involved being seated during the grounding/sham-grounding sessions on days 1 and 2 for 4 hours with grounding patches attached to the front of both quadriceps and with their feet on a grounding pad on the floor. Instructions, set up, grounding time, and break-down time took approximately 4 hours and 15 minutes. Total time per subject for all 4 days was very close to 8 hours 47 minutes.

\section{Muscle soreness}

Using the VAS that is commonly used to assess muscle soreness, ${ }^{18}$ the intensity of the perceived soreness of the quadriceps muscles was assessed. A $20 \mathrm{~cm}$ VAS was used with the left extreme labeled as "no quadriceps soreness" and the right extreme labeled as "maximum quadriceps soreness". In an earlier experiment, ${ }^{13}$ the same scale was used and it correlated with a pressure tolerance measurement taken on the eccentrically damaged calf muscles.

\section{Muscular damage}

CK enzyme leakage from a damaged muscle has been repeatedly used as a marker of muscular damage. ${ }^{19}$ 


\section{White blood cells}

In two previous studies relating to eccentric contractions and grounding, one published ${ }^{13}$ and one unpublished (Brown R. Unpublished data, 2013), there were indications that differences in neutrophils between grounded and sham-grounded subjects existed. In the original pilot study, ${ }^{13}$ neutrophils between groups showed greater than $10 \%$ difference. In an unpublished 2013 study (Brown R. Unpublished data, 2013) differences in neutrophils did not reach significance level ( $P=0.06$ ), but it was felt that checking them in this study may prove worthwhile.

Since a CBC was being collected, we also looked at red blood cell count, hemoglobin, hematocrit, eosinophils, monocytes, basophils, and platelets. This allowed for comparisons with the results of the pilot study. ${ }^{13}$

\section{Statistical analyses}

A test-retest design was used for this project. All statistical calculations were performed with the NCSS/PASS 2000 Dawson edition statistical software. When the data was normally distributed, $t$-tests were performed: paired $t$-tests within a group and two-sample $t$-tests between groups. When between-group variances were found to be unequal, the Aspin-Welch unequal-variance test was used. When the data was not normally distributed, the Wilcoxon signed-rank test for difference in means was used within a group and the Wilcoxon rank-sum test for difference in means was used between groups. Since we expected an increase in inflammation, pain, and white blood cell markers for both groups (CK, pain, neutrophils, white blood cells, lymphocytes, monocytes, eosinophils, basophils), a one-tailed test was used to compare mean values of days 2, 3, and 4 to day 1 for these parameters. Additionally, a one-tailed test was used to compare mean values of day 2 to day 4 between groups for these parameters since we expected a higher increase for the sham-grounded group compared to the grounded group. For the two parameters where the groups where statistically different at day 1 (red blood cells and hematocrit), a normalization procedure was performed rendering day 1 values similar.

\section{Results}

\section{Muscle soreness}

Both groups of subjects started with a low level of pain as determined by the VAS (1.52 for the sham-grounded group and 1.02 for the grounded group). This may be due to the fact that they were an active group of people and the time off requested before coming to the testing center was not enough to reduce soreness in the body completely. In any case, both experienced significantly more pain after the eccentric knee bends. Although the pain decreased in both groups after day 2 , it continued to be significantly higher than pre-eccentric contractions (Table 2 and Figure 1). All withingroup statistical tests compared day 1 with days 2, 3, and 4, and between-group statistical tests compared days 1-4 of the sham-grounded group with the same days of the grounded group (ie, day 1 sham-grounded with day 1 grounded, etc).

\section{Muscle damage}

The moderate eccentric contractions produced moderate muscle damage. In Table 3 and Figure 2 it can be seen that the CK significantly increased 24 hours after the eccentric contractions in the sham-grounded subjects $(P<0.01)$. However, $\mathrm{CK}$ did not increase significantly in the grounded subjects $(P=0.14)$. On day 2 , there was a significant difference between the sham-grounded and the grounded groups $(P=0.04)$. On day 3 , both groups had CK below their preexercise levels $(P<0.01)$.

\section{CBCs}

\section{Total white blood cells}

There were no significant differences within groups or between groups in total white blood cells (normal range $4.0-10.8 \mathrm{~K} / \mu \mathrm{L} ;$ Table 4$)$.

\section{Neutrophils}

On day 2, the sham-grounded subjects' neutrophils decreased, but the grounded subjects' neutrophils increased (normal range $1.6-8.3 \mathrm{~K} / \mu \mathrm{L}$ ). The statistical test between groups was not significant $(P=0.06)$. However, the between-group difference on day 3 was significant ( $P=0.05$; Table 5 and Figure 3 ).

\section{Lymphocytes}

There were no significant differences within or between groups in lymphocytes (normal range 1.00-2.90 K/ $\mu \mathrm{L}$; Table 6).

Table 2 Visual analog pain scale $(\mathrm{mm})$

\begin{tabular}{lllll}
\hline Group & Day I & Day 2 & Day 3 & Day 4 \\
\hline Sham-grounded & $\mathrm{I} .52$ & 5.73 & 2.47 & 4.94 \\
SD & $\mathrm{I} .32$ & $\mathrm{I} .83$ & $\mathrm{I} .46$ & 2.04 \\
Within group & & $P<0.0 \mathrm{I}^{*}$ & $P=0.02^{*}$ & $P<0.0 \mathrm{I}^{*}$ \\
Grounded & $\mathrm{I} .02$ & 6.00 & 3.44 & 4.69 \\
SD & 0.80 & 2.04 & 2.28 & 2.57 \\
Within group & & $P<0.0 \mathrm{I}^{*}$ & $P<0.0 \mathrm{I}^{*}$ & $P<0.0 \mathrm{I}^{*}$ \\
Between groups & $P=0.29$ & $P=0.36$ & $P=0.16$ & $P=0.38$ \\
\hline
\end{tabular}

Notes: Within group is the mean comparison within one group comparing day 2 , day 3 , and day 4 to day I. Between groups is the mean comparison between groups for the same day. *Significant mean difference, ie, $P \leq 0.05$.

Abbreviation: SD, standard deviation. 


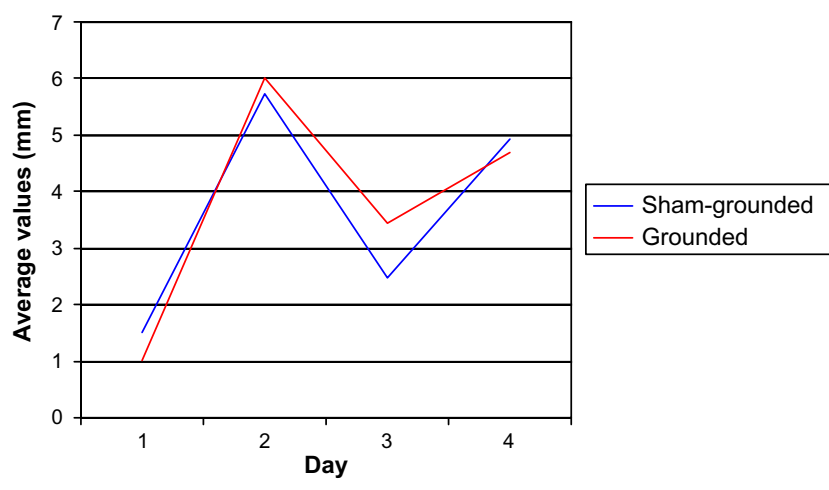

Figure I Visual analog pain scale.

\section{Monocytes}

There were no significant differences within or between groups in monocytes (normal range $0.30-0.80 \mathrm{~K} / \mu \mathrm{L}$; Table 7).

\section{Eosinophils}

On day 3 , there was a significant increase within the grounded group $(P=0.02)$ and a significant difference between groups $(P=0.02)$ as eosinophils increased more in the grounded group than in the sham-grounded group (normal range $0.00-0.20 \mathrm{~K} / \mu \mathrm{L}$; Table 8 and Figure 4).

\section{Basophils}

There were no significant differences within or between groups in basophils (normal range $0.00-0.10 \mathrm{~K} / \mu \mathrm{L}$; Table 9).

\section{Red blood cells}

There were no significant red blood cell differences between groups (normal range 4.7-6.1 M/ $\mu \mathrm{L}$ ). On day 4, there was a significant increase within the grounded group $(P=0.01$; Table 10 and Figure 5). There was no difference between pre and post eccentric contractions for the sham-grounded group.

\section{Hemoglobin}

There were no significant hemoglobin differences between groups (normal range $13.8-16.6 \mathrm{~g} / \mathrm{dL}$ ). On day 3 and day 4 ,

Table 3 Creatine kinase (IU/L)

\begin{tabular}{lllll}
\hline Group & Day I & Day 2 & Day 3 & Day 4 \\
\hline Sham-grounded & 183 & 247 & $\mid 42$ & 159 \\
SD & 90 & $\mid 26$ & $6 \mid$ & 82 \\
Within group & & $P<0.0 I^{*}$ & $P<0.0 I^{*}$ & $P=0.14$ \\
Grounded & 159 & $|7|$ & $\mid 18$ & 132 \\
SD & 1.33 & $|1|$ & 57 & 64 \\
Within group & & $P=0.14$ & $P<0.0 I^{*}$ & $P=0.25$ \\
Between groups & $P=0.09$ & $P=0.04^{*}$ & $P=0.09$ & $P=0.12$ \\
\hline
\end{tabular}

Notes: Within group is the mean comparison within one group comparing day 2 , day 3 , and day 4 to day I. Between groups is the mean comparison between groups for the same day. *Significant mean difference, ie, $P \leq 0.05$.

Abbreviation: SD, standard deviation.

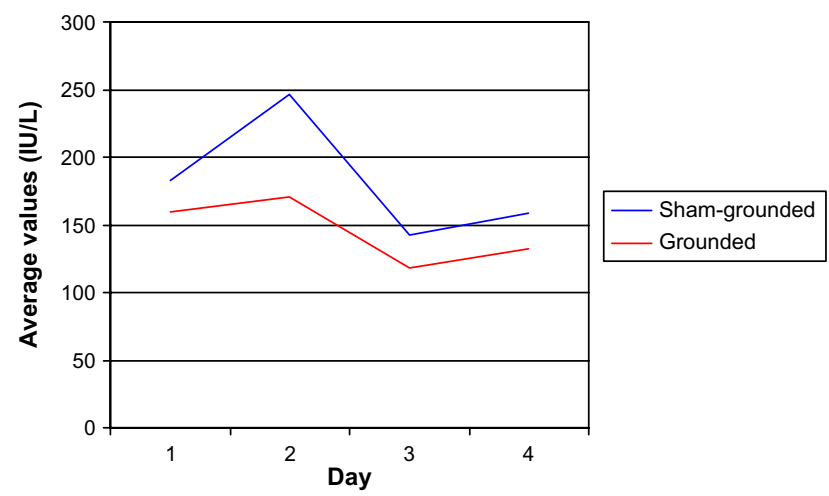

Figure 2 Creatine kinase levels.

Table 4 White blood cells $(\mathrm{K} / \mu \mathrm{L})$

\begin{tabular}{lllll}
\hline Group & Day I & Day 2 & Day 3 & Day 4 \\
\hline Sham-grounded & 6.42 & 6.03 & 6.38 & 6.64 \\
SD & 2.54 & 1.28 & 1.94 & 2.29 \\
Within group & & $P=0.16$ & $P=0.16$ & $P=0.14$ \\
Grounded & 6.76 & 7.33 & 6.87 & 6.88 \\
SD & 1.36 & 3.82 & 1.37 & 1.02 \\
Within group & & $P=0.48$ & $P=0.30$ & $P=0.36$ \\
Between groups & $P=0.15$ & $P=0.13$ & $P=0.09$ & $P=0.14$ \\
\hline
\end{tabular}

Notes: Within group is the mean comparison within one group comparing day 2 , day 3 , and day 4 to day I. Between groups is the mean comparison between groups for the same day.

Abbreviation: SD, standard deviation.

Table 5 Neutrophils $(\mathrm{K} / \mu \mathrm{L})$

\begin{tabular}{lllll}
\hline Group & Day I & Day 2 & Day 3 & Day 4 \\
\hline Sham-grounded & 3.44 & 2.93 & 3.21 & 3.56 \\
SD & 2.47 & 0.91 & 2.04 & 2.09 \\
Within group & & $P=0.13$ & $P=0.45$ & $P=0.1$ I \\
Grounded & 3.47 & 4.01 & 3.40 & 3.64 \\
SD & 0.95 & 2.76 & 0.88 & 0.84 \\
Within group & & $P=0.30$ & $P=0.44$ & $P=0.18$ \\
Between groups & $P=0.09$ & $P=0.06$ & $P=0.05^{*}$ & $P=0.12$ \\
\hline
\end{tabular}

Notes: Within group is the mean comparison within one group comparing day 2 day 3 , and day 4 to day I. Between groups is the mean comparison between groups for the same day. *Significant mean difference, ie, $P \leq 0.05$.

Abbreviation: SD, standard deviation.

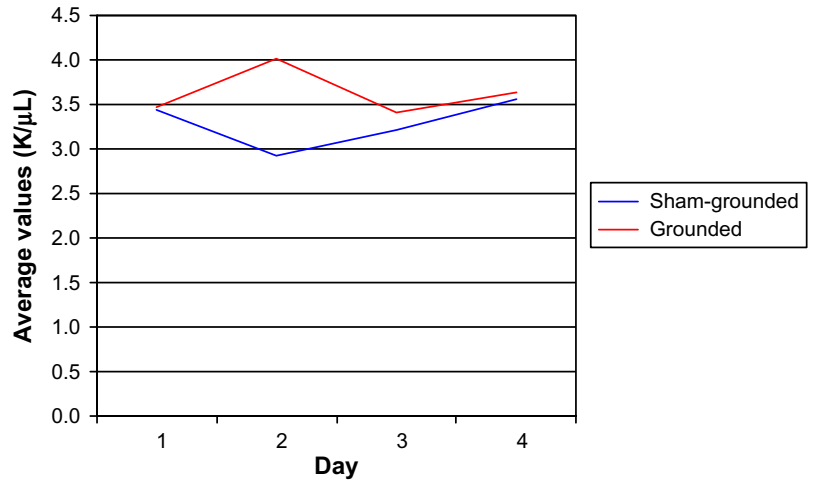

Figure 3 Neutrophils. 
Table 6 Lymphocytes $(\mathrm{K} / \mu \mathrm{L})$

\begin{tabular}{lllll}
\hline Group & Day I & Day 2 & Day 3 & Day 4 \\
\hline Sham-grounded & 2.23 & 2.37 & 2.38 & 2.26 \\
SD & 0.49 & 0.50 & 0.84 & 0.64 \\
Within group & & $P=0.06$ & $P=0.19$ & $P=0.49$ \\
Grounded & 2.39 & 2.46 & 2.54 & 2.37 \\
SD & 0.61 & $I .02$ & 0.81 & 0.77 \\
Within group & & $P=0.29$ & $P=0.16$ & $P=0.42$ \\
Between groups & $P=0.40$ & $P=0.42$ & $P=0.27$ & $P=0.35$ \\
\hline
\end{tabular}

Notes: Within group is the mean comparison within one group comparing day 2 , day 3 , and day 4 to day I. Between groups is the mean comparison between groups for the same day.

Abbreviation: SD, standard deviation.

Table 7 Monocytes $(\mathrm{K} / \mu \mathrm{L})$

\begin{tabular}{lllll}
\hline Group & Day I & Day 2 & Day 3 & Day 4 \\
\hline Sham-grounded & 0.57 & 0.55 & 0.59 & 0.63 \\
SD & 0.20 & 0.14 & 0.17 & 0.23 \\
Within group & & $P=0.35$ & $P=0.18$ & $P=0.07$ \\
Grounded & 0.66 & 0.67 & 0.66 & 0.65 \\
SD & 0.23 & 0.38 & 0.15 & 0.18 \\
Within group & & $P=0.20$ & $P=0.31$ & $P=0.45$ \\
Between groups & $P=0.26$ & $P=0.15$ & $P=0.09$ & $P=0.25$ \\
\hline
\end{tabular}

Notes: Within group is the mean comparison within one group comparing day 2 , day 3 , and day 4 to day I. Between groups is the mean comparison between groups for the same day.

Abbreviation: SD, standard deviation.

Table 8 Eosinophils $(K / \mu L)$

\begin{tabular}{lllll}
\hline Group & Day I & Day 2 & Day 3 & Day 4 \\
\hline Sham-grounded & 0.14 & 0.15 & 0.16 & 0.15 \\
SD & 0.06 & 0.07 & 0.13 & 0.10 \\
Within group & & $P=0.18$ & $P=0.33$ & $P=0.47$ \\
Grounded & 0.20 & 0.19 & 0.23 & 0.19 \\
SD & 0.11 & 0.09 & 0.10 & 0.10 \\
Within group & & $P=0.14$ & $P=0.02^{*}$ & $P=0.14$ \\
Between groups & $P=0.07$ & $P=0.16$ & $P=0.02^{*}$ & $P=0.11$ \\
\hline
\end{tabular}

Notes: Within group is the mean comparison within one group comparing day 2 , day 3 , and day 4 to day I. Between groups is the mean comparison between groups for the same day. $*$ Significant mean difference, ie, $P \leq 0.05$.

Abbreviation: SD, standard deviation.

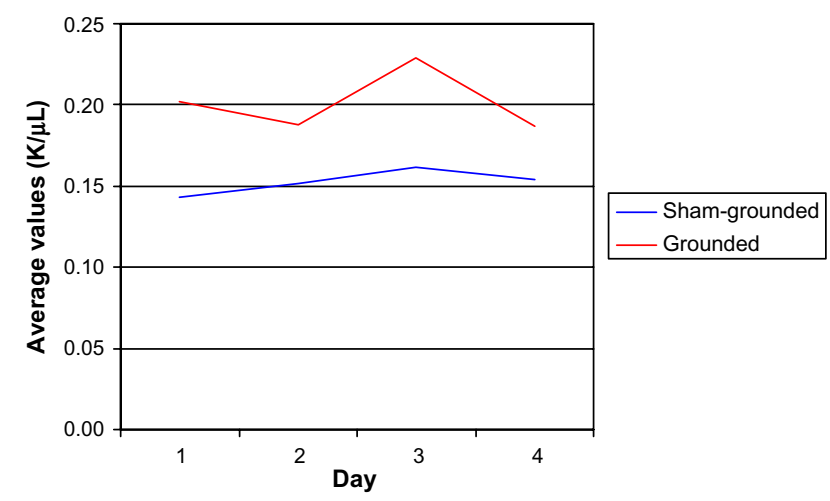

Figure 4 Eosinophils.
Table 9 Basophils $(\mathrm{K} / \mu \mathrm{L})$

\begin{tabular}{lllll}
\hline Group & Day I & Day 2 & Day 3 & Day 4 \\
\hline Sham-grounded & 0.035 & 0.038 & 0.039 & 0.035 \\
SD & 0.017 & 0.019 & 0.035 & 0.018 \\
Within group & & $P=0.19$ & $P=0.32$ & $P=0.50$ \\
Grounded & 0.034 & 0.030 & 0.033 & 0.032 \\
SD & 0.14 & 0.010 & 0.014 & 0.015 \\
Within group & & $P=0.14$ & $P=0.44$ & $P=0.31$ \\
Between groups & $P=0.41$ & $P=0.21$ & $P=0.49$ & $P=0.30$ \\
\hline
\end{tabular}

Notes: Within group is the mean comparison within one group comparing day 2 , day 3 , and day 4 to day I. Between groups is the mean comparison between groups for the same day.

Abbreviation: SD, standard deviation.

there were significant increases within the grounded group ( $P=0.02$ and $P=0.01$, respectively). There was no difference between pre and post for the sham-grounded group (Table 11 and Figure 6).

\section{Hematocrit}

There were no significant hematocrit differences between groups (normal range $42.9 \%-49.1 \%$ ). On day 3 and day 4 , there were significant increases within the grounded group ( $P=0.05$ and $P=0.01$, respectively; Table 12 and Figure 7). There was no difference between pre and post days for the sham-grounded group.

\section{Red cell distribution width}

There were no significant differences within or between groups in red cell distribution width (normal range $11.5 \%-14.5 \%$; Table 13 ).

\section{Bilirubin}

There were no significant differences within or between groups in bilirubin (normal range $0.3-1.0 \mathrm{mg} / \mathrm{dL}$; Table 14).

Table 10 Red blood cells $(M / \mu L)$

\begin{tabular}{lllll}
\hline Group & Day I & Day 2 & Day 3 & Day 4 \\
\hline Sham-grounded & 5.12 & 5.10 & 5.14 & 5.14 \\
SD & 0.33 & 0.35 & 0.32 & 0.25 \\
Within group & & $P=0.28$ & $P=0.27$ & $P=0.38$ \\
Grounded & 5.12 & 5.11 & 5.17 & 5.24 \\
SD & 0.21 & 0.26 & 0.28 & 0.27 \\
Within group & & $P=0.43$ & $P=0.08$ & $P=0.01 *$ \\
Between groups & $P=0.50$ & $P=0.47$ & $P=0.38$ & $P=0.13$ \\
\hline
\end{tabular}

Notes: Within group is the mean comparison within one group comparing day 2 , day 3 , and day 4 to day I. Between groups is the mean comparison between groups for the same day. *Significant mean difference, ie, $P \leq 0.05$.

Abbreviation: SD, standard deviation. 


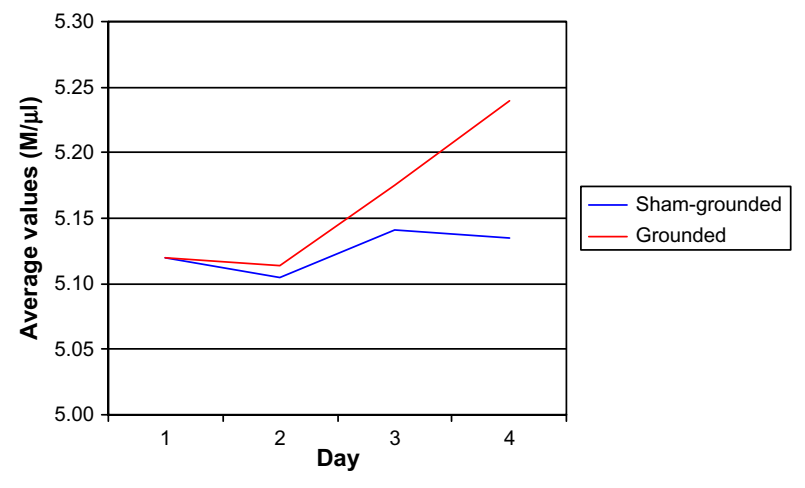

Figure 5 Red blood cells.

Table I I Hemoglobin $(g / d L)$

\begin{tabular}{lllll}
\hline Group & Day I & Day 2 & Day 3 & Day 4 \\
\hline Sham-grounded & 15.39 & 15.46 & 15.50 & 15.36 \\
SD & 0.99 & $I .06$ & 0.93 & 0.77 \\
Within group & & $P=0.2 \mathrm{I}$ & $P=0.14$ & $P=0.42$ \\
Grounded & 14.83 & $|4.9|$ & $\mid 5.04$ & 15.14 \\
SD & 0.72 & 0.89 & 0.75 & 0.83 \\
Within group & & $P=0.12$ & $P=0.02 *$ & $P=0.01 *$ \\
Between groups & $P=0.07$ & $P=0.06$ & $P=0.07$ & $P=0.22$ \\
\hline
\end{tabular}

Notes: Within group is the mean comparison within one group comparing day 2 day 3 , and day 4 to day I. Between groups is the mean comparison between groups for the same day. *Significant mean difference, ie, $P \leq 0.05$.

Abbreviation: SD, standard deviation.

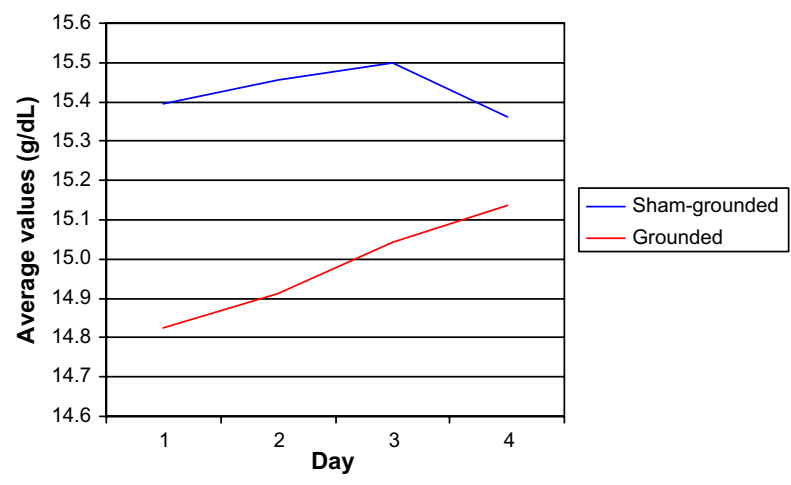

Figure 6 Hemoglobin.

Table I 2 Hematocrit (\%)

\begin{tabular}{lllll}
\hline Group & Day I & Day 2 & Day 3 & Day 4 \\
\hline Sham-grounded & 45.19 & 44.98 & 45.45 & 45.28 \\
SD & 2.44 & 2.43 & 2.35 & 1.66 \\
Within group & & $P=0.12$ & $P=0.20$ & $P=0.41$ \\
Grounded & 45.19 & 45.17 & 45.79 & 46.10 \\
SD & 1.53 & 2.00 & 2.20 & 2.12 \\
Within group & & $P=0.49$ & $P=0.05^{*}$ & $P=0.01 *$ \\
Between groups & $P=0.50$ & $P=0.41$ & $P=0.34$ & $P=0.12$ \\
\hline
\end{tabular}

Notes: Within group is the mean comparison within one group comparing day 2 , day 3 , and day 4 to day I. Between groups is the mean comparison between groups for the same day. *Significant mean difference, ie, $P \leq 0.05$.

Abbreviation: SD, standard deviation.

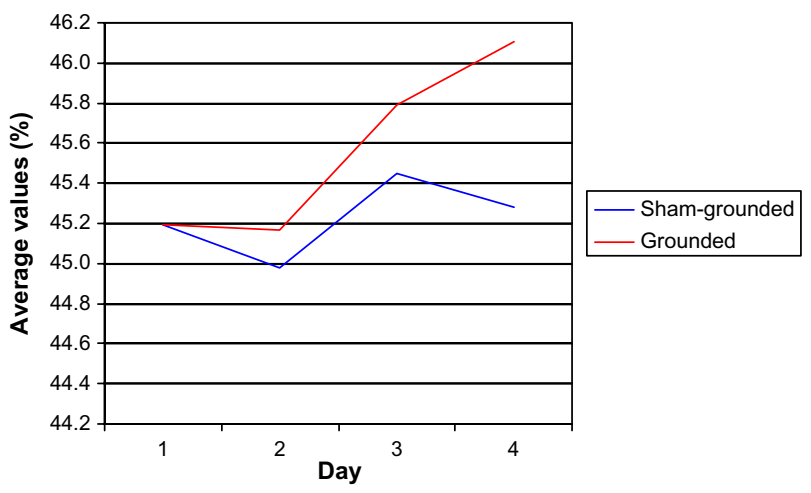

Figure 7 Hematocrit.

\section{Platelets}

There were no significant platelet differences within groups (normal range 130-400 K/ $\mu \mathrm{L}$ ). However, there were significant platelet differences between groups on day $2(P=0.04)$, day $3(P=0.04)$, and day $4(P=0.03)$ with the grounded group having significant increases in platelets in their blood (Table 15 and Figure 8).

\section{Discussion}

Concentric contractions cause muscles to shorten while eccentric contractions cause muscles to lengthen due to the high external load. ${ }^{20}$ There is an association between eccentric contractions and muscle damage. ${ }^{14}$ Eccentric contractions, compared to concentric contractions, cause greater disruptions of muscle cells and their membranes. ${ }^{21-23}$

Following eccentric contractions, skeletal muscle fibers suffer damage including myofilament disorganization, membrane disruption, ${ }^{15}$ sarcomere damage, Z-line streaming, ${ }^{16,24}$ degradation of muscle protein, leakage of muscular enzymes into the plasma, ${ }^{25}$ and degradation of calcium-sensitive pathways..$^{15,26-30}$

The injuries to the muscle cells result in a condition called DOMS. ${ }^{16}$ DOMS is a normal response to eccentric

Table I 3 Red cell distribution width (\%)

\begin{tabular}{lllll}
\hline Group & Day I & Day 2 & Day 3 & Day 4 \\
\hline Sham-grounded & 12.68 & 12.68 & 12.66 & 12.65 \\
SD & 0.63 & 0.64 & 0.64 & 0.64 \\
Within group & & $P=0.44$ & $P=0.35$ & $P=0.21$ \\
Grounded & 12.69 & 12.71 & 12.69 & 12.66 \\
SD & 0.75 & 0.73 & 0.82 & 0.75 \\
Within group & & $P=0.32$ & $P=0.42$ & $P=0.13$ \\
Between groups & $P=0.94$ & $P=0.46$ & $P=0.46$ & $P=0.43$ \\
\hline
\end{tabular}

Notes: Within group is the mean comparison within one group comparing day 2 , day 3 , and day 4 to day I. Between groups is the mean comparison between groups for the same day.

Abbreviation: SD, standard deviation. 
Table 14 Bilirubin (mg/dL)

\begin{tabular}{lllll}
\hline Group & Day I & Day 2 & Day 3 & Day 4 \\
\hline Sham-grounded & 0.87 & 0.89 & 0.92 & 0.88 \\
SD & 0.43 & 0.53 & 0.69 & 0.57 \\
Within group & & $P=0.27$ & $P=0.46$ & $P=0.34$ \\
Grounded & 0.80 & 0.88 & 0.76 & 0.83 \\
SD & 0.39 & 0.43 & 0.39 & 0.46 \\
Within group & & $P=0.07$ & $P=0.09$ & $P=0.37$ \\
Between groups & $P=0.68$ & $P=0.39$ & $P=0.30$ & $P=0.48$
\end{tabular}

Notes: Within group is the mean comparison within one group comparing day 2 , day 3 , and day 4 to day I. Between groups is the mean comparison between groups for the same day.

Abbreviation: SD, standard deviation.

contractions and usually occurs within 24 hours after the activity. ${ }^{31,32}$ The severity of the discomfort depends upon the duration and intensity of the activity. ${ }^{26,33}$

The structural damage leads to an inflammatory response which increases histamines, which sensitize free nerve endings in the muscle tissue and cause pain and swelling. ${ }^{15,31,32}$ The inflammatory response also attracts neutrophils to the injury site where they generate free radicals that participate in the immune response but can also further damage the muscle cells. ${ }^{31,32}$

\section{Muscle soreness}

The pain due to the injury is transmitted via group IV afferent nerve fibers that end in connective tissue between fibers. ${ }^{26}$ The pain can begin as early as 8 hours after the eccentric contractions, peaks between 24 and 72 hours, and is usually

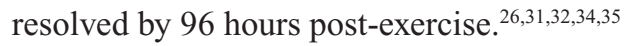

In the present study, both groups experienced moderate levels of pain on days 2, 3, and 4. This result is different than the pain results presented in a pilot study where it was consistently seen that pain level perception was higher for the placebo group. ${ }^{13}$ However, the muscles injured were different (gastrocnemius vs quadriceps) and the intensity of the eccentric contractions in the pilot study were more intense.

Table I 5 Platelets $(\mathrm{K} / \mu \mathrm{L})$

\begin{tabular}{lllll}
\hline Group & Day I & Day 2 & Day 3 & Day 4 \\
\hline Sham-grounded & 220 & 219 & 218 & 217 \\
SD & 40 & 36 & 37 & 38 \\
Within group & & $P=0.45$ & $P=0.34$ & $P=0.28$ \\
Grounded & 238 & 244 & 240 & 241 \\
SD & 33 & 40 & 34 & 29 \\
Within group & & $P=0.11$ & $P=0.26$ & $P=0.24$ \\
Between groups & $P=0.16$ & $P=0.04^{*}$ & $P=0.04^{*}$ & $P=0.03^{*}$ \\
\hline
\end{tabular}

Notes: Within group is the mean comparison within one group comparing day 2 , day 3 , and day 4 to day I. Between groups is the mean comparison between groups for the same day. $*$ Significant mean difference, ie, $P \leq 0.05$.

Abbreviation: SD, standard deviation.

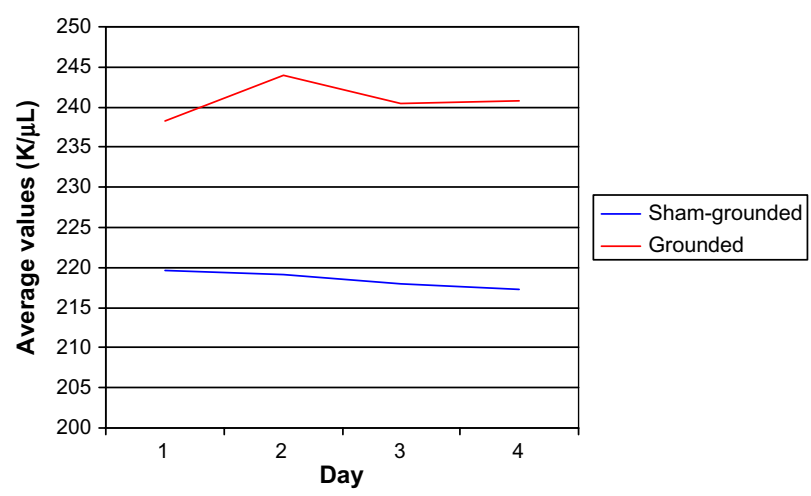

Figure 8 Platelets.

\section{Treatment}

External therapies to reduce pain and improve healing have proved of little value. Stretching, acupuncture, hyperbaric oxygen, laser therapy, ultrasound, pulsed electric current, and transcutaneous nerve stimulation have all been tried with no success. ${ }^{26} \mathrm{~A}$ yoga session after the eccentric contractions increased peak muscle soreness. ${ }^{26}$ Cryotherapy, ice-water baths, and ice massage provided temporary relief and no improvement in healing. ${ }^{26}$

Non-steroidal anti-inflammatory drugs and other general analgesics also fail to provide relief or healing and have no influence on the inflammatory response to eccentric contractions. ${ }^{26,30-32,36}$ One review referenced four studies that implied that non-steroidal anti-inflammatory drugs may actually slow repair and regeneration. ${ }^{29}$

In one study, a 30-minute massage was administered to one group of subjects 2 hours after the eccentric contractions while a second group just rested. Neutrophils and CK were determined every 30 -minutes for 8 hours after the massage. DOMS and $\mathrm{CK}$ were reduced in the massage group $(P<0.05)$ while neutrophils showed a prolonged elevation. The reason for this was not understood. ${ }^{37}$

Other therapies showed some promise but need more studies. These included Farabloc (an electromagnetic shield), body vibration, chemical moist heat, tart cherry juice that reduced strength loss from $22 \%$ to $4 \%$, topical menthol, and branched-chain amino acids. ${ }^{26}$

One study found a difference in DOMS pain using the 1 to 10 VAS. A two-channel, as opposed to a one-channel, frequency-specific microcurrent was used as a treatment after eccentric contractions. After taking readings at 24, 48, and 72 hours post-exercise, the treated group had an average VAS score of 1.01 and the sham-treated group had an average VAS score of 5.40. At each of the three points in time, the difference was significant $(P=0.0005){ }^{38}$ 


\section{CK}

For well over 20 years, eccentric contractions and, as a result, the elevation of CK have been a popular area of study. ${ }^{20,39}$ Significant amounts of $\mathrm{CK}$ are found where energy demands are high. CK is involved in the regeneration of phosphocreatine in the production of energy. It is closely bound to the sarcoplasmic reticulum and sarcomeres of myofibrils. ${ }^{21}$

The increase of CK in the blood after eccentric exercise is connected with cell membrane integrity and mechanical damage of muscle fibers. ${ }^{23}$ This increase is considered an indicator of muscle damage. ${ }^{16,21,26,39}$

Sex, age, individual variability, and training levels can produce different levels of $\mathrm{CK}$ for the same eccentric exercise. Another variable is the intensity of the eccentric contractions that produces corresponding levels of $\mathrm{CK}$ in the blood. ${ }^{21}$ The more intense the exercise, the higher the CK and the longer delay in the CK peak. ${ }^{21,23,25,30,40,41}$ Peak soreness can occur between 24 and 96 hours after the eccentric con tractions. . $^{14,21,27,36,37,42,43}$

The range of $\mathrm{CK}$ in the blood varies widely and depends on various factors, especially the intensity of the activity and the sex of the person. The normal range of $\mathrm{CK}$ for young healthy men is usually $55-170 \mathrm{IU} / \mathrm{L}$.

Many studies have shown significantly higher CK after eccentric exercise. In these studies, the range has varied from pretest values of 83 units/L to 154 units/L; after 24 hours from 230 to 822 units/L; after 48 hours from 20 to 598 units/L; after 72 hours from 200 to 1,620 units/L; and after 96 hours from 132 to 500 units/L..$^{15,21,23,25,42,44}$ Two studies recorded peak CK levels of over 10,000 units/L. ${ }^{16,39}$ In one training session in 1980, completed by Athletics West world-class runners who were asked to do 100 sit-ups with a $11.3 \mathrm{~kg}$ (25 pounds) weight on their chests, CK levels were recorded near 30,000 units/L. The major point is that in all studies, reviewed CK levels rose significantly.

A magnetic resonance imaging study showed that eccentric contractions and the resulting damage caused changes in signal intensity. ${ }^{43}$ In a magnetic resonance spectroscopy study, $\mathrm{Pi} / \mathrm{PCr}$ increased by $31 \%$ and inorganic phosphate/ATP ratio increased by $55 \%$ over 24 hours. ${ }^{34}$ Other molecules that can be detected by magnetic resonance spectroscopy showed a response to eccentric contractions. Erythrocyte-reduced glutathione concentration decreased by $23 \%$ by 24 hours and at the same time CK increased. ${ }^{44}$

In a 2010 study, ${ }^{13}$ the $\mathrm{Pi} / \mathrm{PCr}$ of ungrounded subjects increased by an average of $4.25 \%$ over 72 hours while the $\mathrm{Pi} / \mathrm{PCr}$ of the grounded subjects decreased by an average of
$12.47 \%$. This is a difference of almost $17 \%$ between groups and reinforces a trend indicating a potential advantage for grounding.

In the present study, the subjects who were sham-grounded experienced a significant increase in CK within the group for day 2 and day $3(P<0.01)$ while the grounded subjects did not experience a significant increase for day $2(P=0.14)$ and experienced a significant decrease for day $3(P<0.01)$. The $\mathrm{CK}$ difference between groups was significant for day $2(P=0.04)$ and CK remained higher for the sham-grounded group for days 2-4 (Table 3). This result is similar to the result obtained in the pilot study. ${ }^{13}$ The CK was able to show significant group difference on day 2 while the VAS did not. The CK is objective and more subtle than the VAS which is subjective. The CK can pick up small differences in muscle damage that a subject may not feel. The eccentric contractions were only moderate and did not produce the extreme discomfort that a more extensive protocol would elicit.

\section{White blood cells}

White blood cells mount an inflammatory defense against conditions that threaten normal function and increase in the blood following eccentric contractions. ${ }^{16,29}$

In the present study, there were no significant differences between groups in total white blood cells (Table 4). This result is different from that obtained in the pilot study where sham-grounded subjects had increases while the grounded group experienced decreases. ${ }^{13}$

\section{Neutrophils}

Neutrophils are the first type of white blood cells to enter injured muscle tissue. ${ }^{16,29,35,42}$ At rest, many neutrophils are connected to the endothelial walls of blood vessels. At the onset of eccentric contractions, increases in cell-signaling molecules disconnect the neutrophils from the vessel walls, resulting in mobilization into the circulation. ${ }^{29}$ Then, substances released from injured muscle tissue attract neutrophils to the injury site, initiating inflammation and releasing ROS. ${ }^{16,29,33,45-48}$ Cellular cytokines are also stimulated to attract more proinflammatory proteins. ${ }^{16,21,26,30,35,41,46,48-50}$ The cytokine stimulation also reaches the bone marrow where more neutrophils are put into the circulation for migration to the injured tissue. ${ }^{29}$

As with $\mathrm{CK}$, the circulating neutrophil count is greater depending upon the intensity of the eccentric contractions $^{29,30,42,45}$ and individual characteristics of the subjects. ${ }^{39}$

Additional neutrophil increases are stimulated within minutes post-eccentric contractions and studies have shown 
that they continue to increase for several hours. ${ }^{26,35,42,45,46}$ By 24 hours, the circulating neutrophil count tends to return to near normal. However, they have been seen to remain elevated for as long as 5 days. ${ }^{30,35,41,47}$

The initial invasion of neutrophils is often followed by a secondary invasion as the process switches from proinflammatory to anti-inflammatory. ${ }^{29,41,45,50}$ This secondary invasion results from bone marrow release of neutrophils in response to elevated blood catecholamine levels and cytokine stimulation. However, the neutrophils of the secondary invasion appear to produce more ROS than the first group of neutrophils and are possibly responsible for additional damage. ${ }^{16,28,35,41,46,48}$ The production of ROS released by the neutrophils initially aids in healing, but secondary neutrophils and the ROS they produce may be a double-edged sword because they can contribute to further tissue injury and cell membrane damage. ${ }^{16,35,41,42,51}$

All this suggests that damage and repair may be related. ${ }^{41}$ The dual roles of neutrophil invasion create a paradox for medical personnel. Should clinicians interfere with the secondary damage, limiting inflammation, or is it a part of the healing process? ${ }^{29}$ The major question is, "do neutrophils stimulate healing or contribute to damage?" The answer appears to be both.

In the present study, the grounded group had an increase in neutrophils on day 2 while the sham-grounded group had a decrease. On day 3 , both groups came almost back to the day 1 value, but the grounded group was much closer to the day 1 value and the between-group difference was significant $(P=0.05$; Table 5$)$. This is different from the pilot study where there was always a greater increase in neutrophils for the sham-grounded group. ${ }^{13}$ This result reinforces the question with respect to the role of the first and secondary invasion of neutrophils and the role of grounding in the process.

\section{Eosinophils}

Eosinophils are found in relatively low numbers within the blood. They are multifunctional leukocytes involved in tissue homeostasis, modulation of adaptive immune responses, and innate immunity to certain microbes..$^{52,53}$ In the present study, we found that there was a significant increase for the grounded group at day $3(P=0.02$; Table 8$)$ resulting in a significantly higher cell count for the grounded group compared to the sham-grounded group on that day $(P=0.02)$. This result is different than that obtained in the pilot project were eosinophils were lower for the grounded group at days 2,3 , and $4 .{ }^{13}$

\section{Red blood cells, hemoglobin, and hematocrit}

Additional red blood cells are seen in extracellular spaces as a result of the tissue damage. ${ }^{26}$ On day 4 , the red blood cells increased significantly in the grounded group $(P=0.01$; Table 10). On days 3 and 4 , the hemoglobin and hematocrit (Tables 11 and 12) were also significantly higher in the grounded group as might be expected due to the increase in red blood cells (hemoglobin day $3 P=0.02$ and day $4 P=0.01$; hematocrit day $3 P=0.05$ and day $4 P=0.01$ ). This result is different than the result obtained in the pilot project where no red blood cell marker showed significant changes. ${ }^{13}$

\section{Platelets}

Platelets are the second most abundant cells, after red blood cells, in the blood circulation. ${ }^{54} \mathrm{~A}$ major physiological role of platelets is to accumulate at sites of damaged blood vessels and initiate the blood clotting process. ${ }^{54}$

Recent studies have revealed that platelets do not just plug the leak in damaged blood vessels; they are also key components in inflammation and immune responses. ${ }^{22,54} \mathrm{As}$ early responders to injury sites, they are well placed to aid in the immune response. ${ }^{22}$ Platelets contain many chemokines, a small family of cytokines that recruit additional platelets and immune cells, especially neutrophils, to the site of infection or injury. ${ }^{49,54}$

Platelet activating factor (PAF) is activated and elevated by eccentric exercise. ${ }^{33}$ PAF is a glycerol ether phospholipid inflammatory mediator involved in the immune response. . $^{27,33,54}$ There is a positive relationship between PAF, eccentric exercise-induced muscle damage, and the damage marker CK. Neutrophils also increase after exposure to PAF. ${ }^{33}$

Platelets contain both proinflammatory cytokines and growth factors, including basic fibroblast growth factor and anti-inflammatory cytokines. ${ }^{49,54}$ These cytokines may be selectively released depending on local inflammation status, progression of the healing process, the site of the injury, and the cellular environment. ${ }^{49,54}$

As can be seen from Table 15, the grounded subjects had significantly higher levels of platelets than the sham-grounded subjects on days $2(P=0.04), 3(P=0.04)$, and $4(P=0.03)$. Again, this result is different than the result reported in the pilot study where the grounded group had a consistently lower mean platelet volume than the sham-grounded group. ${ }^{13}$

\section{ROS/free radicals}

ROS, or free radicals, are entities with an unpaired electron. They are highly reactive and, because they steal electrons 
from other molecules, can start a dangerous chain reaction that can damage important cellular components like DNA.

To prevent the potential damage, the body has a defense system of antioxidants that can provide an electron to free radicals and stop the chain reaction before damage occurs. Along with enzyme systems in the body that scavenge free radicals, there are also dietary nutrients that act as antioxidants.

As with neutrophils, the picture is still unclear regarding the possibility that ROS promote both injury and repair. ${ }^{55}$

Evidence is available that specific free radicals - nitric oxide and superoxide - are released by skeletal muscle tissue. These free radicals help promote the invasion of neutrophils, but can release more ROS via a respiratory burst. ${ }^{33,41,51,55,56}$

Free radicals are a necessary part of the healing process. Nitric oxide regulates repair of injured skeletal muscle. Its activation, beginning with nitric oxide production on endothelial cells, facilitates blood flow to injured cells. ${ }^{45,55,57}$ Muscle-derived nitric oxide helps regulate neutrophilmediated lysis of muscle cells and scavenges another free radical, superoxide. ${ }^{55}$ Superoxide by itself is a mild oxidant but it can be converted by other free radicals to hydrogen peroxide, which is a stronger oxidant that can also damage cell membranes and be converted to an even more reactive free radical, peroxynitite. ${ }^{35,55}$ Myeloperoxidase is expressed by neutrophils and converts hydrogen peroxide to water. The amount of myeloperoxidase can determine whether superoxide production leads to the reduced production of more powerful free radicals..$^{55,58}$

Free radical production is affected by exercise and injured muscle tissue. It increases their cytolytic capacity. The extent to which free radicals promote further muscle injury is determined by the history of muscle use, the intensity of the use, and the numbers and state of activation of the invading neutrophils. ${ }^{55}$ Free radicals are found in injured muscle tissue 4 hours after damage occurs and, if the free radicals are over-activated, they have both a direct and indirect role in muscle damage. ${ }^{35,41}$

Free radicals released by macrophages in the presence of neutrophils help repair the tissue by allowing phagocytosis of debris by macrophages, but they also can promote muscle damage. ${ }^{55}$

With respect to the high production of free radicals, it might be helpful if, in addition to the body's antioxidants and the dietary antioxidants, there could be another source of free electrons to scavenge the free radicals. Free electrons from the earth seem to be able to fulfill this function.

\section{Possible mechanisms}

It has been shown that earthing influences cortisol secretion patterns and sleep quality. ${ }^{11}$ It was also shown that earthing affects mineral and electrolyte concentration in blood serum, especially iron, ionized calcium, inorganic phosphorus, sodium, potassium, and magnesium. Renal excretion of both phosphorus and calcium decreased significantly. Earthing affects thyroid function and secretions, impacting almost every physiological process in the body, and it was shown to accelerate immune response following vaccination. ${ }^{8}$ Earthing was also shown to greatly affect the function of the autonomic nervous system. ${ }^{3,12,59}$ There is also the previous DOMS study showing an impact on white blood cells, bilirubin, $\mathrm{CK}, \mathrm{Pi} / \mathrm{PCr}$, glycerolphosphorylcholine, phosphorylcholine, and pain. ${ }^{13}$

These results suggest that connecting the body to the earth enables free electrons and diurnal electrical rhythms to enter the body, setting the biological clocks for hormones that regulate sleep and activity. ${ }^{17}$ It was also suggested that free electrons from the earth neutralize the positively charged free radicals that are the hallmark of chronic inflammation. ${ }^{60}$ Another possible mechanism of inflammation control is the inflammatory reflex. This neural reflex mechanism regulates innate immune responses and inflammation during tissue injury and pathogen invasion mainly through the vagus nerve. Since grounding stimulates the parasympathetic branch of the autonomic nervous system, it is possible that vagus nerve-mediated cholinergic stimulation produces a decrease in inflammation. ${ }^{59}$

Limitations in time and resources prevented the repetition of the first study at a larger scale. These limitations also prevented measurement of range of motion, which would have given information on function; they also prevented the possibility of using imaging and performing biopsies on damaged muscles. Future studies incorporating these modalities and a larger number of participants with longer grounding periods, and followed for a longer period of time (longitudinal study), would definitely add validity to the results of this study and the previous pilot study.

\section{Conclusion}

While the study showed no difference in pain relief, it demonstrated that grounding after moderate eccentric contractions resulted in differences in certain markers between and/or within groups. The grounded group, on various days, had significantly higher neutrophils and platelets. Practical significance with respect to neutrophils and grounding should be further investigated because of 1) the important role of neutrophils in both proinflammatory and anti-inflammatory 
responses, and 2) the tendency of neutrophils in three previous studies to approach statistical significance. Platelet increase may be important due to their recently discovered participation in the immune inflammatory responses. The sham-grounded group had significantly higher $\mathrm{CK}$ on day 2 while the grounded subjects did not experience an increase in $\mathrm{CK}$. This is an important result because $\mathrm{CK}$ due to eccentric contractions usually increases significantly in both groups. Grounding reduced the loss of CK from the injured muscle cells, indicating a healing effect.

\section{Acknowledgments}

Financial support was provided by Earth FX Inc., Palm Spring, CA, USA, and earthing products were provided by earthing.com, Palm Springs, CA, USA.

\section{Disclosures}

$\mathrm{R}$ Brown is an independent contractor hired by Earth FX for this project. G Chevalier has been an independent contractor for Earth FX since 2007. Earth FX and earthing.com played no role in the study design, data analysis and interpretation, or writing of the manuscript. The authors report no other conflicts of interest.

\section{References}

1. Williams ER, Heckman SJ. The local diurnal variation of cloud electrification and the global diurnal variation of negative charge on the Earth. J Geophys Res. 1993;98(D3):5221-5234.

2. Anisimov SV, Mareev EA, Bakastov SS. On the generation and evolution of aeroelectric structures in the surface layer. J Geophys Res. 1999;104(D12):14359-14367.

3. Chevalier G, Mori K, Oschman JL. The effect of earthing (grounding) on human physiology. European Biology and Bioelectromagnetics. 2006;2(1):600-621.

4. Chevalier G, Sinatra ST, Oschman JL, Delany RM. Earthing (grounding) the human body reduces blood viscosity-a major factor in cardiovascular disease. J Altern Complement Med. 2013;19(2):102-110.

5. Oschman JL. Charge transfer in the living matrix. J Bodyw Mov Ther. 2009;13(3):215-228.

6. Sokal K, Sokal P. Earthing the human organism influences bioelectrical processes. J Altern Complement Med. 2012;18(3):229-234.

7. Applewhite R. The effectiveness of a conductive patch and a conductive bed pad in reducing induced human body voltage via the application of earth ground. European Biology and Bioelectromagnetics. 2005;1: $23-40$.

8. Sokal K, Sokal P. Earthing the human body influences physiologic processes. J Altern Complement Med. 2011;17(4):301-308.

9. Sokal P, Jastrzebski Z, Jaskulska E, et al. Differences in Blood Urea and Creatinine Concentrations in Earthed and Unearthed Subjects during Cycling Exercise and Recovery. Evid Based Complement Alternat Med. 2013;2013:382643.

10. Chevalier G, Sinatra ST. Emotional Stress, Heart Rate Variability, Grounding, and Improved Autonomic Tone: Clinical Applications. Integrative Medicine. 2011;10(3):16-21.

11. Ghaly M, Teplitz D. The biologic effects of grounding the human body during sleep as measured by cortisol levels and subjective reporting of sleep, pain, and stress. J Altern Complement Med. 2004;10(5): 767-776.
12. Chevalier G. Changes in pulse rate, respiratory rate, blood oxygenation, perfusion index, skin conductance, and their variability induced during and after grounding human subjects for 40 minutes. J Altern Complement Med. 2010;16(1):81-87.

13. Brown D, Chevalier G, Hill M. Pilot study on the effect of grounding on delayed-onset muscle soreness. J Altern Complement Med. 2010;16(3): 265-273.

14. Jamurtas AZ, Theocharis V, Tofas, $\mathrm{T}$, et al. Comparison between leg and arm eccentric exercises of the same relative intensity on indices of muscle damage. Eur J Appl Physiol. 2005;95(2-3):179-185.

15. Sudhakar S, Naiya A. Biochemical markers an indirect method for evaluating delayed onset muscle soreness among recreational athletes. Int J Biol Med Res. 2012;3(2):1624-1626.

16. Kenney WL, Wilmore JH, Costill DL, editors. Muscle Soreness and Cramps. In: Physiology of Sport and Exercise. 5th ed. Champaign: Human Kinetics; 2011:237-241.

17. Oschman JL. Can electrons act as antioxidants? A review and commentary. J Altern Complement Med. 2007;13(9):955-967.

18. Revill SI, Robinson JO, Rosen M, Hogg MI. The reliability of a linear analogue for evaluating pain. Anaesthesia. 1976;31(9):1191-1198.

19. Smith LL, Brunetz MH, Chenier TC, et al. The effects of static and ballistic stretching on delayed onset muscle soreness and creatine kinase. Res Q Exerc Sport. 1993;64(1):103-107.

20. Hentzen E, Lahey M, Peters D, et al. Stress-dependent and stress-independent expression of the myogenic regulatory factors and the MARP genes after eccentric contractions. J Physiol. 2006;570(1):157-167.

21. Baird MF, Graham SM, Baker JS, Bickerstaff GF. Creatine-kinase- and exercise-related muscle damage implications for muscle performance and recovery. J Nutr Metab. 2012;2012:960363.

22. Cox D, Kerrigan SW, Watson SP. Platelets and the innate immune system: mechanisms of bacterial-induced platelet activation. J Thromb Haemost. 2011;9(6):1097-1107.

23. Poprzęcki S, Staszkiewicz A, Hübner-Woźniak E. Effect of eccentric and concentric exercise on plasma creatine kinase (CK) and lactate dehydrogenase (LDH) activity in healthy adults. Biol Sport. 2004;21(2):193-203.

24. Fridén J, Sjöström M, Ekblom B. Myofibrillar damage following intense eccentric exercise in man. Int J Sports Med. 1983;4(3):170-176.

25. Newham DJ, Jones DA, Edwards RH. Plasma creatine kinase changes after eccentric and concentric contractions. Muscle Nerve. 1986;9(1): 59-63.

26. Kedlaya D, Lorenzo CT. Postexercise muscle soreness. Medscape; 2014 May 8. Available from: http://emedicine.medscape.com/article/313267overview. Accessed April 27, 2015.

27. Milias GA, Nomikos T, Fragopoulou E, Athanasopoulos S, Antonopoulou S. Effects of eccentric exercise-induced muscle injury on blood levels of platelet activating factor (PAF) and other inflammatory markers. Eur J Appl Physiol. 2005;95(5-6):504-513.

28. Armstrong RB. Initial events in exercise-induced muscular injury. Med Sci Sports Exerc. 1990;22(4):429-435.

29. Butterfield TA, Best TM, Merrick MA. The dual roles of neutrophils and macrophages in inflammation: a critical balance between tissue damage and repair. J Athl Train. 2006;41(4):457-465.

30. Peake J, Nosaka K, Suzuki K. Characterization of inflammatory responses to eccentric exercise in humans. Exerc Immunol Rev. 2005; 11 : 64-85.

31. Connolly DA, Sayers SP, McHugh MP. Treatment and prevention of delayed onset muscle soreness. J Strength Cond Res. 2003;17(1): 197-208.

32. Szymanski DJ. Recommendations for the avoidance of delayed-onset muscle soreness. Strength Cond J. 2001;23(4):7-13.

33. Green SJ, Mellouk S, Hoffman SL, Meltzer MS, Nacy CA. Cellular mechanisms of nonspecific immunity to intracellular infection: cytokine-induced synthesis of toxic nitrogen oxides from L-arginine by macrophages and hepatocytes. Immunol Lett. 1990;25(1-3):15-19.

34. Lund H, Vestergaard-Poulsen P, Kanstrup IL, Sejrsen, P. Isokinetic eccentric exercise as a model to induce and reproduce pathophysiological alterations related to delayed onset muscle soreness. Scand J Med Sci Sports. 1998;8(4):208-215. 
35. Kanda K, Sugama K, Hayashida H, et al. Eccentric exercise-induced delayed-onset muscle soreness and changes in markers of muscle damage and inflammation. Exerc Immunol Rev. 2013;19:72-85.

36. Tokmakidis SP, Kokkinidis EA, Smilios I, Douda H. The effects of ibuprofen on delayed muscle soreness and muscular performance after eccentric exercise. J Strength Cond Res. 2003;17(1):53-59.

37. Smith LL, Keating MN, Holbert D, et al. The effects of athletic massage on delayed onset muscle soreness, creatine kinase, and neutrophil count: a preliminary report. J Orthop Sports Phys Ther. 1994;19(2):93-99.

38. Curtis D, Fallows S, Morris M, McMakin C. The efficacy of frequency specific microcurrent therapy on delayed onset muscle soreness. J Bodyw Mov Ther. 2010;14(3):272-279.

39. Clarkson PM, Kearns AK, Rouzier P, Rubin R, Thompson PD. Serum creatine kinase levels and renal function measures in exertional muscle damage. Med Sci Sports Exerc. 2006;38(4):623-627.

40. Melin B, Bourdon L, Jimenez C, Charpenet A, Bernard O. Plasma myosin and creatine kinase time-course after a concentric-eccentric field exercise. Arch Physiol Biochem. 1997;105(1):27-31.

41. Toumi H, F'guyer S, Best TM. The role of neutrophils in injury and repair following muscle stretch. J Anat. 2006;208(4):459-470.

42. Smith LL, Semple SJ, McKune AJ, et al. Changes in neutrophil count, creatine kinases and muscle soreness after repeated bouts of downhill running. South African Journal of Sports Medicine. 2007;19(3): 86-93.

43. Sorichter S, Mair J, Koller A, et al. Creatine kinase, myosin heavy chains and magnetic resonance imaging after eccentric exercise. J Sports Sci. 2001;19(9):687-691.

44. Lee J, Goldfarb AH, Rescino MH, Hegde S, Patrick S, Apperson K. Eccentric exercise effect on blood oxidative-stress markers and delayed onset of muscle soreness. Med Sci Sports Exerc. 2002;34(3): 443-448.

45. Cannon JG, St Pierre BA. Cytokines in exertion-induced skeletal muscle injury. Mol Cell Biochem. 1998;179(1-2):159-167.

46. Filippin LI, Moreira AJ, Marroni NP, Xavier RM. Nitric oxide and repair of skeletal muscle injury. Nitric Oxide. 2009;21(3-4):157-163.

47. Haslett C. Resolution of acute inflammation and the role of apoptosis in the tissue fate of granulocytes. Clin Sci (Lond). 1992;83(6):639-648.
48. Tidball JG. Inflammatory cell response to acute muscle injury. Med Sci Sports Exerc. 1995;27(7):1022-1032.

49. Middleton KK, Barro V, Muller B, Terada S, Fu FH. Evaluation of the effects of platelet-rich plasma (PRP) therapy involved in the healing of sports-related soft tissue injuries. Iowa Orthop J. 2012;32:150-163.

50. Smith C, Kruger MJ, Smith DM, Myburgh KH. The inflammatory response to skeletal muscle injury: illuminating complexities. Sports Med. 2008;38(11):947-969.

51. Pizza FX, Peterson JM, Baas JH, Koh TJ. Neutrophils contribute to muscle injury and impair its resolution after lengthening contractions in mice. J Physiol. 2005;562(Pt 3):899-913.

52. Kita H. Eosinophils: multifaceted biologic properties and roles in health and disease. Immunol Rev. 2011;242(1):161-177.

53. Shamri R, Xenakis JJ, Spencer LA. Eosinophils in innate immunity: an evolving story. Cell Tissue Res. 2011;343(1):57-83.

54. Li C, Li J, LiY, et al. Crosstalk between platelets and the immune system: old systems with new discoveries. Adv Hematol. 2012; 2012:384685.

55. Lobo V, Patil A, Phatak A, Chandra N. Free radicals, antioxidants and functional foods: Impact on human health. Pharmacogn Rev. 2010;4(8):118-126.

56. Brickson S, Ji LL, Schell K, Olabisi R, St Pierre Schneider B, Best TM. M1/70 attenuates blood-borne neutrophil oxidants, activation, and myofiber damage following stretch injury. J Appl Physiol (1985). 2003;95(3):969-976.

57. Filippin LI, Cuevas MJ, Lima E, Marroni NP, Gonzalez-Gallego J, Xavier RM. Nitric oxide regulates the repair of injured skeletal muscle. Nitric Oxide. 2011;24(1):43-49.

58. Pulli B, Ali M, Forghani R, et al. Measuring myeloperoxidase activity in biological samples. PLoS One. 2013;8(7):e67976.

59. Chevalier G. Grounding the human body improves facial blood flow regulation: results of a randomized, placebo controlled pilot study. Journal of Cosmetics, Dermatological Sciences and Applications. 2014;4:293-308.

60. Oschman JL, Chevalier G, Brown R. The effects of grounding (earthing) on inflammation, the immune response, wound healing, and prevention and treatment of chronic inflammatory and autoimmune diseases. J Inflamm Res. 2015;8:83-96.
Open Access Journal of Sports Medicine

\section{Publish your work in this journal}

Open Access Journal of Sports Medicine is an international, peer-reviewed, open access journal publishing original research, reports, reviews and commentaries on all areas of sports medicine. The manuscript management system is completely online and includes a very quick and fair peer-review system.

\section{Dovepress}

Visit http://www.dovepress.com/testimonials.php to read real quotes from published authors. 